PAPER

\title{
Gaussian Process Regression for Prediction of Electric Power Damage Caused by Typhoons Considering Nonstationarity of Damage
}

\author{
Tomohiro Hachino, Tatsuya Ueda and Hitoshi Takata \\ Department of Electrical and Electronics Engineering, Kagoshima University \\ 1-21-40 Korimoto, Kagoshima 890-0065, Japan \\ E-mail: hachino@eee.kagoshima-u.ac.jp
}

\begin{abstract}
Electric power systems in Japan suffer from major damage from typhoons almost every year. Typhoons often cause undesirable long-time power failures. To speedily restore the electric power supply, one needs to predict the amount of damage accurately for an approaching typhoon. This paper presents a method of predicting the amount of electric power damage for the Amami archipelago using Gaussian process (GP) regression. The typhoon track is evaluated by considering its wind characteristics. A nonstationary kernel is introduced as the covariance function of the GP in consideration of the nonstationarity of damage. This predictor can yield not only the predicted amounts of damage but also their confidence measures. Simulation results based on actual data are shown to illustrate the effectiveness of this approach.
\end{abstract}

Keywords: prediction, damage by typhoon, electric power system, Gaussian process regression, nonstationary kernel

\section{Introduction}

Damage to electric power facilities is caused by typhoons, which are one of the most common meteorological disasters in Japan [1],[2]. The power supply is sometimes cut off in wide areas for a long time by typhoons, having an undesirable effect on society. To ensure the speedy restoration of the electric power supply, it is important to predict the amount of damage accurately for an approaching typhoon. For island areas such as the Amami archipelago, developing an accurate prediction method for electric power damage is particularly urgent. This is because the staff and materials necessary for restoration must be appropriately arranged and sent to isolated islands according to the predicted amounts of damage before the arrival of the typhoon.

So far, rough predictions based on experience have been made in the field using past typhoon weather information and electric power damage. On the other hand, the authors have presented two-stage predictors that consist of neural networks and linear or second-order regression from the viewpoint of nonlinear prediction [3]-[5]. However, these prediction methods require a large number of parameters to describe the nonlinearity between the typhoon weather information and the electric power damage. This is presently one of the drawbacks of these predictors, because we can use only limited amounts of training input (typhoon weather information) and output (electric power damage) data. Moreover, confidence measures for the predicted amount of damage cannot be obtained for the two-stage predictors. To overcome these problems, we proposed a nonparametric predictor based on the Gaussian process (GP) model [6]. The GP model is a nonparametric model and fits naturally into the Bayesian framework [7]-[9]. This model has recently attracted much attention for system identification [10],[11], time series forecasting [12]-[14], and predictive control [15]. The GP-based predictor includes far fewer parameters to describe the nonlinearity than the two-stage predictors, and moreover it can give not only predicted amounts of damage but also their confidence measures. This predictor utilizes a stationary kernel as the prior covariance function of the GP. Since the relation between the typhoon weather information and the amount of electrical power damage is in general nonstationary, the accuracy of prediction can be greatly influenced by the use of the nonstationary covariance function.

To improve the accuracy of prediction, in this paper, we present a modified prediction method for the Amami archipelago using GP regression. A nonstationary kernel is introduced as the covariance function of the GP in consideration of the nonstationarity of the amount of damage. The quantification of the 
typhoon track is crucially important for prediction. Thus, here we take account of the characteristics of typhoons as follows. The wind force in the east side of a typhoon is stronger than that in the west side of the typhoon. Also, the wind force in the center of the typhoon is quite weak [16]. Therefore, in this paper, we propose the use of a sum of two Gaussian functions to quantify the typhoon track considering the wind characteristics of the typhoon.

This paper is organized as follows. In Sect. 2, the problem is formulated. In Sect. 3, the quantification technique for the typhoon track is given using the sum of two Gaussian functions. In Sect. 4, the modified GP prior model based on the nonstationary covariance function is derived and its training algorithm is presented using particle swarm optimization (PSO). In Sect. 5, the estimation method of the typhoon weather data used for prediction is described. In Sect. 6, the amount of electric power damage is predicted from the GP posterior distribution. In Sect. 7, the performance of the proposed prediction method is demonstrated through numerical simulation using actual data. Finally, conclusions are given in Sect. 8 .

\section{Statement of the Problem}

The input of the predictor is the typhoon weather information:

$$
\boldsymbol{x}=\left[x_{1}, x_{2}\right]^{\mathrm{T}}
$$

where $x_{1}$ is the typhoon track and $x_{2}[\mathrm{~m} / \mathrm{s}]$ is the maximum instantaneous wind speed. The output from the predictor is the amount of electric power damage $y$, such as the number of circuits with power failure. It is possible to choose other weather information as the input, but this increases the scale of the predictor. Therefore, we choose only the typhoon track and the maximum instantaneous wind speed as information greatly affecting the amount of electric power damage.

It is assumed that we collect the typhoon weather data released from Japan Meteorological Agency:

$$
\begin{gathered}
\boldsymbol{X}=[\boldsymbol{x}(1), \boldsymbol{x}(2), \cdots, \boldsymbol{x}(N)]^{\mathrm{T}} \\
\boldsymbol{x}(j)=\left[x_{1}(j), x_{2}(j)\right]^{\mathrm{T}}
\end{gathered}
$$

and the corresponding actual data of the amount of electric power damage:

$$
\boldsymbol{y}=[y(1), y(2), \cdots, y(N)]^{\mathrm{T}}
$$

where $N$ is the number of the typhoons that hit or came close to the Amami archipelago in the past.

The purpose of this paper is to construct a prediction system that can predict the amount of electric power damage with its confidence measure from the weather data of a new approaching typhoon.

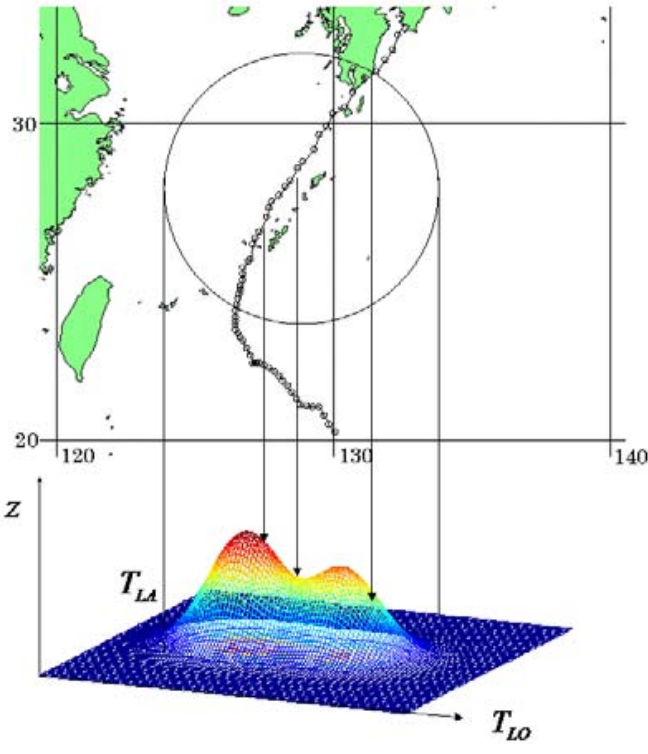

Fig. 1 Quantification of the typhoon track

\section{Quantification of Typhoon Track}

The typhoon track strongly correlates with the amount of electric power damage. In order to input the typhoon track into the predictor, we have to quantify it as a numerical value. In general, in the Northern Hemisphere, the wind force in the east side of a typhoon is stronger than that in the west side of the typhoon, and the wind force in the center of the typhoon is quite weak [16]. These wind characteristics suggest that a typhoon passing by the west side of the Amami archipelago causes more damage than a typhoon passing by the east side of the Amami archipelago. Moreover, since a typhoon is likely to stay near the Amami archipelago for a long time, the electric power system may frequently suffer from major damage. Therefore, we have to consider the wind characteristics and the stagnancy of the typhoon when the typhoon track is quantified.

We introduce the following quantification technique for the typhoon track. First, the centers of the typhoon are plotted every hour in the range from latitude $26^{\circ} \mathrm{N}$ to $31^{\circ} \mathrm{N}$. Then, the sum of two Gaussian functions is superimposed on the Amami archipelago as shown in Fig. 1. The numerical value of the typhoon track is calculated by summing the altitude values of the arranged function corresponding to the plotted centers as follows:

$$
\begin{aligned}
x_{1}= & \sum_{j=1}^{n} z_{j} \\
z_{j}= & \gamma \exp \left\{-\frac{\left(T_{L A j}-C_{L A}\right)^{2}+\left(T_{L O j}-C_{L O}+\alpha_{1}\right)^{2}}{\beta^{2}}\right\} \\
& +\exp \left\{-\frac{\left(T_{L A j}-C_{L A}\right)^{2}+\left(T_{L O j}-C_{L O}-\alpha_{2}\right)^{2}}{\beta^{2}}\right\}
\end{aligned}
$$


where $T_{L A j}$ is the latitude of the typhoon center, $T_{L O j}$ is the longitude of the typhoon center, $C_{L A}$ is the latitude of the Amami archipelago, $C_{L O}$ is the longitude of the Amami archipelago, $\alpha_{1}>0$ and $\alpha_{2}>0$ are the biases for the typhoon center, $\beta$ is the width of the Gaussian function, $\gamma>1$ is the amplitude of the western Gaussian function, and $n$ is the number of plotted centers of the typhoon. Note that the value of the typhoon track increases in the case that the typhoon stays near the Amami archipelago for a long time. The sum of two Gaussian functions is introduced to describe the wind characteristics of the typhoon mentioned above. A way of determining the adjustment parameters $\alpha_{1}, \alpha_{2}, \beta$, and $\gamma$ suboptimally will be discussed in Sect. 4.2.

\section{GP Prior Regression}

\subsection{Derivation of GP prior model}

Assume that the relation between the typhoon weather information $\boldsymbol{x}$ and the amount of electric power damage $y$ is described as

$$
y=f(\boldsymbol{x})+\varepsilon
$$

where $f(\cdot)$ is a function which is assumed to be smooth. $\varepsilon$ is assumed to be a zero-mean Gaussian noise with variance $\sigma_{n}^{2}$. The assumption of smoothness means that the amounts of electric power damage have a high correlation and become similar values for the typhoon weather data that are close to each other. The determination of the standard deviation $\sigma_{n}$ is discussed in Sect. 4.2 and Appendix.

Let the function value vector corresponding to the typhoon weather data given by Eq. (2) be

$$
\boldsymbol{f}=[f(\boldsymbol{x}(1)), f(\boldsymbol{x}(2)), \cdots, f(\boldsymbol{x}(N))]^{\mathrm{T}}
$$

Then this function value vector $\boldsymbol{f}$ can be represented by GP regression. The GP is a Gaussian random function and is completely described by its mean function and covariance function. We can regard it as a collection of random variables with a joint multivariable Gaussian distribution. Therefore, the function value vector $\boldsymbol{f}$ can be represented by the GP as

$$
\boldsymbol{f} \sim \mathcal{N}(\boldsymbol{m}(\boldsymbol{X}), \boldsymbol{\Sigma}(\boldsymbol{X}, \boldsymbol{X}))
$$

where $\boldsymbol{m}(\boldsymbol{X})$ is the $N$-dimensional mean function vector and $\boldsymbol{\Sigma}(\boldsymbol{X}, \boldsymbol{X})$ is the $N$-dimensional covariance matrix evaluated at all pairs of training input data. Equation (7) means that $\boldsymbol{f}$ has a Gaussian distribution with the mean function vector $\boldsymbol{m}(\boldsymbol{X})$ and the covariance matrix $\boldsymbol{\Sigma}(\boldsymbol{X}, \boldsymbol{X})$.

In this paper, the mean function $m(\boldsymbol{x})$ is expressed as a first-order polynomial, i.e., a linear combination of the input variable:

$$
m(\boldsymbol{x})=\overline{\boldsymbol{x}} \boldsymbol{\theta}_{m}
$$

where $\overline{\boldsymbol{x}}=\left[\boldsymbol{x}^{\mathrm{T}}, 1\right]$ and $\boldsymbol{\theta}_{m}=\left[\theta_{m 1}, \theta_{m 2}, \theta_{m 3}\right]^{\mathrm{T}}$ is the unknown weighting parameter vector for the mean function. Thus, the mean function vector $\boldsymbol{m}(\boldsymbol{X})$ is described as follows:

$$
\begin{aligned}
\boldsymbol{m}(\boldsymbol{X}) & =[m(\boldsymbol{x}(1)), m(\boldsymbol{x}(2)), \cdots, m(\boldsymbol{x}(N))]^{\mathrm{T}} \\
& =\overline{\boldsymbol{X}} \boldsymbol{\theta}_{m}
\end{aligned}
$$

where $\overline{\boldsymbol{X}}=[\boldsymbol{X}, \boldsymbol{e}]$ and $\boldsymbol{e}=[1,1, \cdots, 1]^{\mathrm{T}}$ is the $N$ dimensional vector consisting of ones. As no prior information about the mean function is available for this prediction problem, it is natural to use the first-order polynomial of the input variable as the mean function. This setting does not limit the prediction because the posterior (predictive) mean function is improved to an appropriate nonlinear function by the trained covariance function (see Eq. (21)).

The covariance matrix $\boldsymbol{\Sigma}(\boldsymbol{X}, \boldsymbol{X})$ is constructed as

$$
\boldsymbol{\Sigma}(\boldsymbol{X}, \boldsymbol{X})=\left[\begin{array}{cccc}
\Sigma_{11} & \Sigma_{12} & \cdots & \Sigma_{1 N} \\
\Sigma_{21} & \Sigma_{22} & \cdots & \Sigma_{2 N} \\
\vdots & \vdots & & \vdots \\
\Sigma_{N 1} & \Sigma_{N 2} & \cdots & \Sigma_{N N}
\end{array}\right]
$$

where the element $\Sigma_{p q}=\operatorname{cov}(f(\boldsymbol{x}(p), f(\boldsymbol{x}(q)))=$ $s(\boldsymbol{x}(p), \boldsymbol{x}(q))$ is a function of $\boldsymbol{x}(p)$ and $\boldsymbol{x}(q)$. The following Gaussian kernel is often utilized as the covariance function $s(\boldsymbol{x}(p), \boldsymbol{x}(q))[6]$ :

$$
s(\boldsymbol{x}(p), \boldsymbol{x}(q))=\sigma_{y}^{2} \exp \left(-\frac{\|\boldsymbol{x}(p)-\boldsymbol{x}(q)\|^{2}}{2 \ell^{2}}\right)
$$

where $\|\cdot\|$ denotes the Euclidean norm. This stationary covariance function depends only on the distance between $\boldsymbol{x}(p)$ and $\boldsymbol{x}(q)$, and does not depend on the values of $\boldsymbol{x}(p)$ and $\boldsymbol{x}(q)$ as shown in Fig. 2(a). Actually, in our data, the variance of the amount of electric power damage is 19.8 for a group with a small norm of the weather data, and it is 24.1 for a group with a large norm of the weather data. This fact suggests that the amount of electric power damage caused by a typhoon is nonstationary with respect to the typhoon weather information and its covariance increases with the amount of typhoon weather information. Therefore, in this paper, we introduce the following nonstationary kernel as the covariance function $s(\boldsymbol{x}(p), \boldsymbol{x}(q))$ instead of Eq. (11):

$$
\begin{aligned}
s(\boldsymbol{x}(p), \boldsymbol{x}(q))= & \sigma_{y}^{2} \exp \left(-\frac{\|\boldsymbol{x}(p)-\boldsymbol{x}(q)\|^{2}}{2 \ell^{2}}\right) \\
& +\sum_{i=1}^{2} v_{i}^{2} x_{i}(p) x_{i}(q)
\end{aligned}
$$

This function is shown in Fig. 2(b). By using this nonstationary covariance function, we can realize the phenomenon that the covariance of the amount of electric power damage increases with the amount of typhoon weather information. A similar nonstationary 


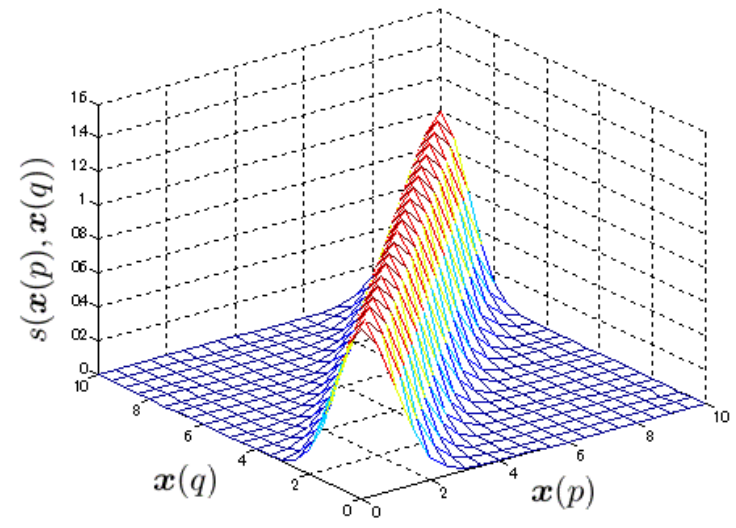

(a) Stationary covariance function

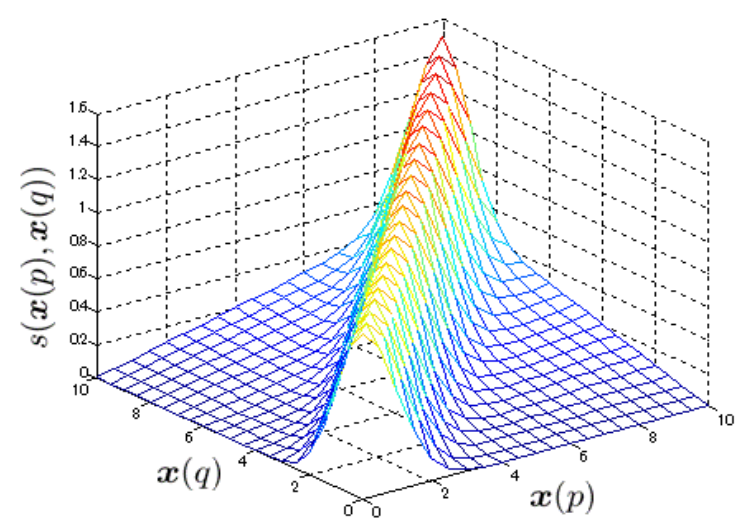

(b) Nonstationary covariance function

Fig. 2 Covariance functions

covariance function to Eq. (12) has been successfully utilized for time series prediction [13].

As the amount of electric power damage $\boldsymbol{y}$ is a noisy observation, we can derive the following GP prior regression from Eq. (7):

$$
\boldsymbol{y} \sim \mathcal{N}(\boldsymbol{m}(\boldsymbol{X}), \boldsymbol{K}(\boldsymbol{X}, \boldsymbol{X}))
$$

where

$$
\begin{aligned}
& \boldsymbol{K}(\boldsymbol{X}, \boldsymbol{X})=\boldsymbol{\Sigma}(\boldsymbol{X}, \boldsymbol{X})+\sigma_{n}^{2} \boldsymbol{I}_{N} \\
& \boldsymbol{I}_{N}: N \times N \text { identity matrix }
\end{aligned}
$$

and $\boldsymbol{\theta}_{c}=\left[\sigma_{y}, \ell, v_{1}, v_{2}, \sigma_{n}\right]^{\mathrm{T}}$ is called the hyperparameter vector. In the following, $\boldsymbol{K}(\boldsymbol{X}, \boldsymbol{X})$ is written as $\boldsymbol{K}$ for simplicity.

\subsection{Training of GP prior model}

The accuracy of the prediction greatly depends on the unknown parameter vectors, i.e., the weighting parameter vector $\boldsymbol{\theta}_{m}=\left[\theta_{m 1}, \theta_{m 2}, \theta_{m 3}\right]^{\mathrm{T}}$ of the mean function, the hyperparameter vector $\boldsymbol{\theta}_{c}=$ $\left[\sigma_{y}, \ell, v_{1}, v_{2}, \sigma_{n}\right]^{\mathrm{T}}$ of the covariance function, and the adjustment parameter vector $\boldsymbol{\theta}_{p}=\left[\alpha_{1}, \alpha_{2}, \beta, \gamma\right]^{\mathrm{T}}$ used for the quantification of the typhoon track. Therefore, the parameter vector $\boldsymbol{\theta}=\left[\boldsymbol{\theta}_{m}^{\mathrm{T}}, \boldsymbol{\theta}_{c}^{\mathrm{T}}, \boldsymbol{\theta}_{p}^{\mathrm{T}}\right]^{\mathrm{T}}$ has to be determined suboptimally. This training is carried out by minimizing the negative log marginal likelihood of the typhoon weather data and the actual amount of electric power damage:

$$
\begin{aligned}
J= & -\log p(\boldsymbol{y} \mid \boldsymbol{X}, \boldsymbol{\theta}) \\
= & \frac{1}{2} \log |\boldsymbol{K}|+\frac{1}{2}(\boldsymbol{y}-\boldsymbol{m}(\boldsymbol{X}))^{\mathrm{T}} \boldsymbol{K}^{-1} \\
& \times(\boldsymbol{y}-\boldsymbol{m}(\boldsymbol{X}))+\frac{N}{2} \log (2 \pi) \\
= & \frac{1}{2} \log |\boldsymbol{K}|+\frac{1}{2}\left(\boldsymbol{y}-\overline{\boldsymbol{X}} \boldsymbol{\theta}_{m}\right)^{\mathrm{T}} \boldsymbol{K}^{-1}\left(\boldsymbol{y}-\overline{\boldsymbol{X}} \boldsymbol{\theta}_{m}\right) \\
& +\frac{N}{2} \log (2 \pi)
\end{aligned}
$$

As the cost function $J$ generally has multiple local minima, this training problem becomes a nonlinear optimization problem. However, we can separate the linear optimization part and the nonlinear optimization part for this problem. Note that if the candidates for the hyperparameter vector $\boldsymbol{\theta}_{c}$ and adjustment parameter vector $\boldsymbol{\theta}_{p}$ are given, the weighting parameter vector $\boldsymbol{\theta}_{m}$ can be estimated by the linear least-squares (LS) method putting $\partial J / \partial \boldsymbol{\theta}_{m}=\mathbf{0}$ :

$$
\boldsymbol{\theta}_{m}=\left(\overline{\boldsymbol{X}}^{\mathrm{T}} \boldsymbol{K}^{-1} \overline{\boldsymbol{X}}\right)^{-1} \overline{\boldsymbol{X}}^{\mathrm{T}} \boldsymbol{K}^{-1} \boldsymbol{y}
$$

However, even if $\boldsymbol{\theta}_{m}$ is known, the optimization with respect to $\boldsymbol{\theta}_{c}$ and $\boldsymbol{\theta}_{p}$ is a complicated nonlinear problem and might suffer from the local minima problem. Therefore, in this paper, we combine the linear LS method with PSO [17] to determine the unknown parameter vector $\boldsymbol{\theta}$. Only $\boldsymbol{\Omega}=\left[\boldsymbol{\theta}_{c}^{\mathrm{T}}, \boldsymbol{\theta}_{p}^{\mathrm{T}}\right]^{\mathrm{T}}$ is represented with the particles and is searched for by PSO. The detailed training algorithm is shown in Appendix.

\section{Estimation of Typhoon Weather Data}

For island areas such as the Amami archipelago, the amount of electric power damage caused by a typhoon is appropriately predicted and the staff and materials necessary for restoration are dispatched to the isolated islands just before ships and airplanes are canceled. In this paper, when the typhoon reaches latitude $23^{\circ} \mathrm{N}$, the prediction of the amount of electric power damage is started. The reason why this timing is chosen for the prediction is that it takes about 24 hours on average for typhoons to reach the Amami archipelago after they reach latitude $23^{\circ} \mathrm{N}$, which gives us just enough time to arrange the staff and materials necessary for restoration in the Amami archipelago. In the following, the time when the typhoon reaches latitude $23^{\circ} \mathrm{N}$ is referred to as the implementation time.

In order to carry out the prediction at the implementation time, the typhoon weather data $\boldsymbol{x}_{*}=$ $\left[x_{1 *}, x_{2 *}\right]^{\mathrm{T}}$ at the time when it comes close to the 
Amami archipelago are necessary. The typhoon weather data are estimated using the predicted typhoon weather data announced by Japan Meteorological Agency.

First, assuming that the typhoon will pass through the centers of the probability circles in the track forecast announced by Japan Meteorological Agency, we estimate the numerical value $x_{1 *}$ of the typhoon track by the quantification technique mentioned in Sect. 3 . Since the centers of the probability circles in the track forecast are usually given with intervals of 12 hours by Japan Meteorological Agency, the centers every hour are plotted by interpolation after two centers of probability circles are linked by a straight line, then the numerical value $x_{1 *}$ of the typhoon track is evaluated by Eq. (4).

The maximum instantaneous wind speed $x_{2 *}$ is estimated from the following second-order polynomial:

$$
\begin{aligned}
x_{2 *} & =a_{0}+a_{1} u_{1}+a_{2} u_{2}+a_{3} u_{1} u_{2}+a_{4} u_{1}^{2}+a_{5} u_{2}^{2} \\
& =\boldsymbol{z}^{\mathrm{T}} \boldsymbol{\vartheta}
\end{aligned}
$$

where $\boldsymbol{z}=\left[1, u_{1}, u_{2}, u_{1} u_{2}, u_{1}^{2}, u_{2}^{2}\right]^{\mathrm{T}}, \boldsymbol{\vartheta}=\left[a_{0}, a_{1}, a_{2}, a_{3}\right.$, $\left.a_{4}, a_{5}\right]^{\mathrm{T}}, u_{1}$ is the maximum wind speed at the implementation time, and $u_{2}$ is the predicted closest distance between the typhoon center and the Amami archipelago. The unknown parameter vector $\boldsymbol{\vartheta}$ is estimated by the linear LS method based on the past data of the maximum instantaneous wind speed at the Amami archipelago, the maximum wind speed at the implementation time, and the predicted closest distance between the typhoon center and the Amami archipelago.

\section{Prediction by GP Posterior Distribution}

Let the amount of electric power damage corresponding to the estimated typhoon weather data $\boldsymbol{x}_{*}=\left[x_{1 *}, x_{2 *}\right]^{\mathrm{T}}$ be $y_{*}$. Then, we can obtain the joint Gaussian distribution of $\boldsymbol{y}$ and $y_{*}$ under the GP prior as

$$
\begin{aligned}
& {\left[\begin{array}{c}
\boldsymbol{y} \\
y_{*}
\end{array}\right] \sim} \\
& \mathcal{N}\left(\left[\begin{array}{l}
\boldsymbol{m}(\boldsymbol{X}) \\
m\left(\boldsymbol{x}_{*}\right)
\end{array}\right],\left[\begin{array}{cc}
\boldsymbol{K} & \boldsymbol{\Sigma}\left(\boldsymbol{X}, \boldsymbol{x}_{*}\right) \\
\boldsymbol{\Sigma}\left(\boldsymbol{x}_{*}, \boldsymbol{X}\right), & s\left(\boldsymbol{x}_{*}, \boldsymbol{x}_{*}\right)+\sigma_{n}^{2}
\end{array}\right]\right)
\end{aligned}
$$

where $\boldsymbol{\Sigma}\left(\boldsymbol{X}, \boldsymbol{x}_{*}\right)=\boldsymbol{\Sigma}^{\mathrm{T}}\left(\boldsymbol{x}_{*}, \boldsymbol{X}\right)$ is the $N$-dimensional covariance vector evaluated at all pairs of the training input $\boldsymbol{X}$ and the new input $\boldsymbol{x}_{*}$. From the formula for conditioning a joint Gaussian distribution [18], the posterior distribution for $y_{*}$ is obtained as

$$
y_{*} \mid \boldsymbol{X}, \boldsymbol{y}, \boldsymbol{x}_{*} \sim \mathcal{N}\left(\hat{y}_{*}, \hat{\sigma}_{*}^{2}\right)
$$

where $\hat{y}_{*}$ is the predictive mean and $\hat{\sigma}_{*}^{2}$ is the predictive variance, which are given as follows:

$$
\hat{y}_{*}=m\left(\boldsymbol{x}_{*}\right)+\boldsymbol{\Sigma}\left(\boldsymbol{x}_{*}, \boldsymbol{X}\right) \boldsymbol{K}^{-1}(\boldsymbol{y}-\boldsymbol{m}(\boldsymbol{X}))
$$

$\hat{\sigma}_{*}^{2}=s\left(\boldsymbol{x}_{*}, \boldsymbol{x}_{*}\right)-\boldsymbol{\Sigma}\left(\boldsymbol{x}_{*}, \boldsymbol{X}\right) \boldsymbol{K}^{-1} \boldsymbol{\Sigma}\left(\boldsymbol{X}, \boldsymbol{x}_{*}\right)+\sigma_{n}^{2}$

$\hat{y}_{*}$ is the predicted amount of electric power damage caused by the typhoon and $\hat{\sigma}_{*}^{2}$ is utilized as the confidence measure of the predicted amount of damage.

\section{Simulations}

We predict the amount of electric power damage using the actual data of 18 typhoons that hit or came close to the Amami archipelago from 1996 to 2009. This archipelago is located around latitude $27.83^{\circ} \mathrm{N}$ and longitude $128.08^{\circ} \mathrm{E}$. Among the 18 typhoons, 17 typhoons are used for training data and one typhoon is used for prediction data. Namely, we can predict the amount of electric power damage with 18 combinations of training and prediction data. The amount of electric power damage is taken to be the number of circuits with power failure. The design parameters of PSO are chosen as follows:

particle size: $Q=100$

maximum iteration number: $l_{\max }=100$

inertia factor: $w^{l}=w_{\max }-\left(w_{\max }-w_{\min }\right) l / l_{\max }$ $\left(w_{\max }=0.8, w_{\min }=0.4\right)$

acceleration coefficients: $c_{1}=0.7, c_{2}=0.7$

The prediction results obtained by the proposed new method and the previous work [6] in which the stationary GP-based method was used, are shown in Table 1 , where $y_{*}(k)$ is the actual amount of damage, i.e., the actual number of circuits with power failure, and $\hat{y}_{*}(k)$ is the predicted number of circuits with power failure. The absolute error $J_{1}(k)=\mid y_{*}(k)-$ $\hat{y}_{*}(k) \mid$ and the error rate $J_{2}(k)=\left|y_{*}(k)-\hat{y}_{*}(k)\right| / y_{*}(k)$ are also included in this table. This table indicates that the absolute errors and the error rates of the proposed method are improved for 12 of the 18 typhoons compared with those of the previous stationary GP-based method. The average absolute error is 3.98 for the proposed method and 4.60 for the stationary GP-based method. The proposed method achieves a $13.5 \%$ improvement in the absolute error. The average error rate is 0.426 for the proposed method and 0.503 for the stationary GP-based method. The average error rate of the proposed method is $15.3 \%$ smaller than that of the stationary GP-based method. Therefore, we can confirm that introducing the nonstationary covariance function given by Eq. (12) and using the quantification technique for the typhoon track proposed in Sect. 3 considerably improve the accuracy of the prediction.

The prediction results obtained by the proposed new method and the stationary GP-based method are also depicted in Figs. 3 and 4, respectively. In these figures, the circles show the actual number of circuits with power failure, the squares show the predicted number of circuits with power failure, and the shaded areas give the double standard deviation confidence interval (95.5\% confidence region). The actual amounts 
Table 1 Prediction results

\begin{tabular}{|c|c||c|c|c||c|c|c|}
\hline \multirow{2}{*}{$\begin{array}{c}\text { Typhoon } \\
\text { number }\end{array}$} & \multirow{2}{*}{$\begin{array}{c}\text { Actual } \\
\text { damage }\end{array}$} & \multicolumn{3}{|c||}{$\begin{array}{c}\text { Proposed } \\
\text { method }\end{array}$} & \multicolumn{3}{c|}{$\begin{array}{c}\text { Stationary GP-based } \\
\text { method }\end{array}$} \\
\cline { 3 - 8 } & $y_{*}(k)$ & $\hat{y}_{*}(k)$ & $J_{1}(k)$ & $J_{2}(k)$ & $\hat{y}_{*}(k)$ & $J_{1}(k)$ & $J_{2}(k)$ \\
\hline 1 & 5 & 6.38 & 1.38 & 0.276 & 7.39 & 2.39 & 0.478 \\
\hline 2 & 8 & 17.30 & 9.30 & 1.162 & 14.17 & 6.17 & 0.771 \\
\hline 3 & 3 & 4.71 & 1.71 & 0.569 & 5.93 & 2.93 & 0.976 \\
\hline 4 & 2 & 5.37 & 3.37 & 1.686 & 6.87 & 4.87 & 2.436 \\
\hline 5 & 7 & 7.90 & 0.90 & 0.129 & 8.15 & 1.15 & 0.164 \\
\hline 6 & 11 & 7.73 & 3.27 & 0.297 & 8.51 & 2.49 & 0.226 \\
\hline 7 & 9 & 11.85 & 2.85 & 0.316 & 9.70 & 0.70 & 0.078 \\
\hline 8 & 21 & 8.50 & 12.50 & 0.595 & 9.91 & 11.09 & 0.528 \\
\hline 9 & 28 & 18.41 & 9.59 & 0.343 & 16.29 & 11.71 & 0.418 \\
\hline 10 & 27 & 23.58 & 3.42 & 0.127 & 30.03 & 3.03 & 0.112 \\
\hline 11 & 6 & 13.11 & 7.11 & 1.185 & 11.20 & 5.20 & 0.867 \\
\hline 12 & 21 & 21.04 & 0.04 & 0.002 & 19.63 & 1.37 & 0.065 \\
\hline 13 & 30 & 30.16 & 0.16 & 0.005 & 28.77 & 1.23 & 0.041 \\
\hline 14 & 21 & 23.54 & 2.54 & 0.121 & 25.36 & 4.36 & 0.208 \\
\hline 15 & 19 & 14.07 & 4.93 & 0.260 & 12.50 & 6.50 & 0.342 \\
\hline 16 & 10 & 7.32 & 2.68 & 0.268 & 7.09 & 2.91 & 0.291 \\
\hline 17 & 30 & 25.77 & 4.23 & 0.141 & 22.34 & 7.66 & 0.255 \\
\hline 18 & 9 & 7.42 & 1.58 & 0.176 & 1.90 & 7.10 & 0.789 \\
\hline \hline average & - & - & 3.98 & 0.426 & - & 4.60 & 0.503 \\
\hline
\end{tabular}

of damage of 15 typhoons are included in the double standard deviation confidence interval for the stationary GP-based method, whereas the actual amounts of damage of 16 typhoons are included in the interval for the proposed new method. Namely, the probability that the actual amounts of damage are included in the double standard deviation confidence interval is $88.9 \%$ for the proposed new method and $83.3 \%$ for the stationary GP-based method. This indicates that the proposed method can also improve the confidence region.

Here, we compare the accuracy of the proposed method and that of the stationary GP-based method. Note that the proposed method gives not only the predicted values but also their confidence regions. Although the absolute errors and error rates of the proposed method are not improved for 6 typhoons compared with those of the stationary GP-based method, as shown in Table 1, the confidence regions might be improved for these 6 typhoons. The goodness of the prediction result should be judged from the accuracy of both the predicted values and the confidence regions. Unfortunately, the predicted values and confidence regions cannot be evaluated by a single performance index simultaneously, because the true confidence regions, i.e., the true variances of the amount of damage, are unknown. Therefore, after the predicted values were evaluated by the absolute errors and the error rates, the probability that the actual amounts of damage are included in the $95.5 \%$ confidence region was examined to check the accuracy of the confidence region. As a result, we conclude that the overall accuracy of the proposed method is superior to that of the stationary GP-based method.

In the two-stage prediction method [4], confidence measures of the predicted values could not be obtained. On the other hand, the proposed method can give not only the predicted values but also their confidence regions. Therefore, in effect, we can utilize the upper value of the confidence region $\hat{y}_{* \max }=\hat{y}_{*}+2 \hat{\sigma}_{*}$ as the predicted value for the worst case. This suggests that the proposed method can reduce the likelihood that the staff and materials necessary for restoration are lacking in the Amami archipelago. This is also one of the advantages of the proposed method.

\section{Conclusions}

In this paper, a nonparametric prediction method for the amount of electric power damage caused by a typhoon has been proposed using the GP regression model. The new quantification technique for the typhoon track is presented considering the wind characteristics of the typhoon. Moreover, a nonstationary kernel function is introduced as the covariance function of the GP to improve the accuracy of the predic- 


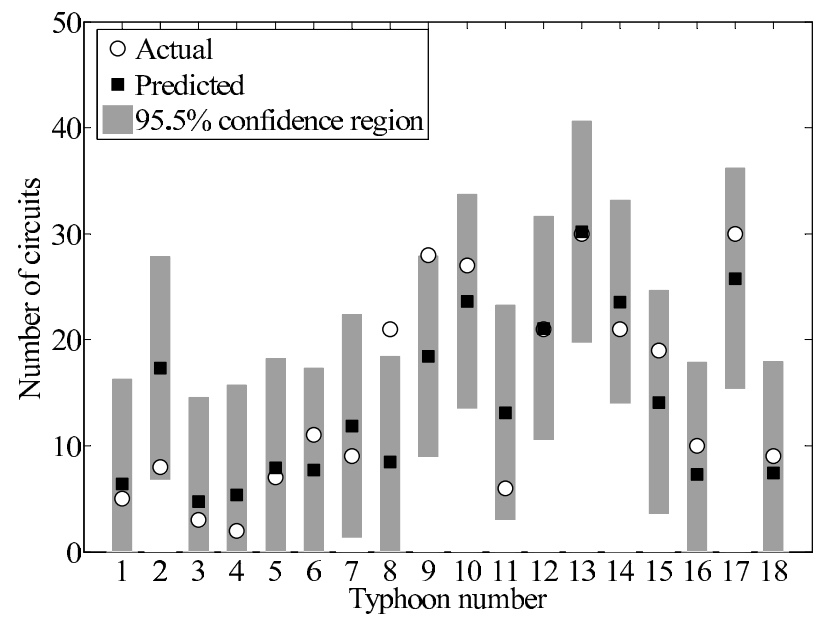

Fig. 3 Predicted number of circuits with power failure (proposed new method)

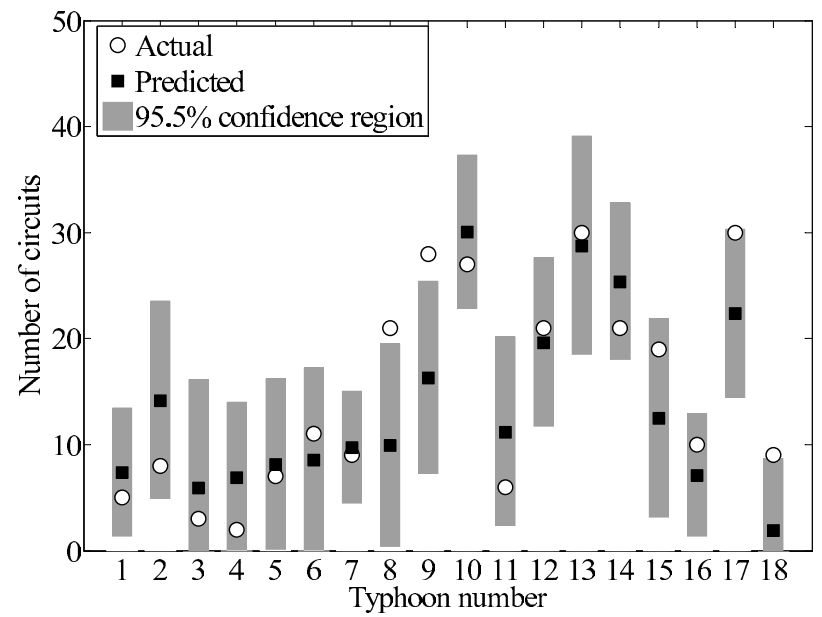

Fig. 4 Predicted number of circuits with power failure (stationary GP-based method)

tion. Simulation results show that the accuracy of the proposed prediction method is superior to that of the conventional prediction method. Compared with the stationary GP-based method, the proposed method achieves a $13.5 \%$ improvement for the absolute error and a $15.3 \%$ improvement for the error rate. The development of the prediction method to take the actual situation of the maintenance of the power system into consideration is planned as a future work.

\section{Appendix}

\section{[PSO Algorithm]}

\section{Step 1: Initialization}

Generate an initial population of $Q$ particles with random positions $\boldsymbol{\Omega}_{[i]}^{0}=\left[\boldsymbol{\theta}_{c[i]}^{\mathrm{T}}, \boldsymbol{\theta}_{p[i]}^{\mathrm{T}}\right]^{\mathrm{T}}$ and velocities
$\boldsymbol{V}_{[i]}^{0}(i=1,2, \cdots, Q)$.

Set the iteration counter $l=0$.

Step 2: Quantification of the typhoon track

Quantify the typhoon track as a numerical value using $\boldsymbol{\theta}_{p[i]}(i=1,2, \cdots, Q)$ by the quantification technique given in Sect. 3 .

Step 3: Construction of the covariance matrix

Construct $Q$ candidates for the covariance matrix $\boldsymbol{K}_{[i]}$ using $\boldsymbol{\theta}_{c[i]}(i=1,2, \cdots, Q)$.

Step 4: Estimation of $\boldsymbol{\theta}_{m}$

Estimate $Q$ candidates for $\boldsymbol{\theta}_{m[i]}(i=1,2, \cdots, Q)$ from Eq. (16).

Step 5: Evaluation value calculation

Calculate the negative log marginal likelihood of the typhoon weather data and the actual amount of electric power damage:

$$
\begin{aligned}
J\left(\boldsymbol{\Omega}_{[i]}^{l}\right)= & -\log p\left(\boldsymbol{y} \mid \boldsymbol{X}_{[i]}, \boldsymbol{\theta}_{[i]}\right) \\
= & \frac{1}{2} \log \left|\boldsymbol{K}_{[i]}\right|+\frac{1}{2}\left(\boldsymbol{y}-\overline{\boldsymbol{X}}_{[i]} \boldsymbol{\theta}_{m[i]}\right)^{\mathrm{T}} \boldsymbol{K}_{[i]}^{-1} \\
& \times\left(\boldsymbol{y}-\overline{\boldsymbol{X}}_{[i]} \boldsymbol{\theta}_{m[i]}\right)+\frac{N}{2} \log (2 \pi)
\end{aligned}
$$

Step 6: Update of the best positions pbest and gbest

Update $\boldsymbol{p b e s t}_{i}^{l}$, which is the personal best position, and $\boldsymbol{g} \boldsymbol{b e s t} \boldsymbol{t}^{l}$, which is the global best position among all particles, as follows:

If $l=0$, then

$$
\begin{aligned}
& \text { pbest }_{[i]}^{l}=\boldsymbol{\Omega}_{[i]}^{l} \\
& \text { gbest }^{l}=\boldsymbol{\Omega}_{\left[i_{\text {best }}\right]}^{l} \quad i_{\text {best }}=\arg \min _{i} J\left(\boldsymbol{\Omega}_{[i]}^{l}\right)
\end{aligned}
$$

otherwise,

$$
\begin{aligned}
& \text { pbest }_{[i]}^{l}= \begin{cases}\boldsymbol{\Omega}_{[i]}^{l} & \left(J\left(\boldsymbol{\Omega}_{[i]}^{l}\right)<J\left(\text { pbest }_{[i]}^{l-1}\right)\right) \\
\text { pbest }_{[i]}^{l-1} & (\text { otherwise })\end{cases}
\end{aligned}
$$

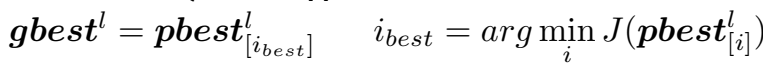

Step 7: Update of positions and velocities

Update the particle positions and velocities using Eq. (25):

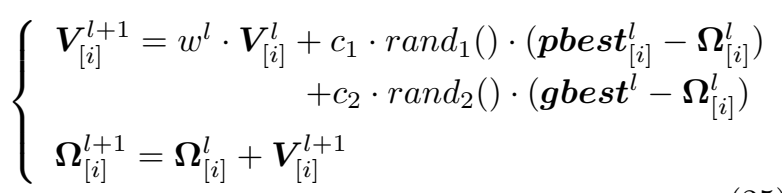

where $w^{l}$ is an inertia factor, $c_{1}$ and $c_{2}$ are constants representing acceleration coefficients, and $\operatorname{rand}_{1}()$ and $\operatorname{rand}_{2}()$ are uniformly distributed random numbers with amplitude in the range $[0,1]$.

Step 8: Repetition

Set the iteration counter to $l=l+1$ and go to Step 2 until the prespecified iteration number $l_{\max }$.

Finally, at the termination of this algorithm when $l=l_{\max }$, the suboptimal $\hat{\boldsymbol{\Omega}}=\left[\hat{\boldsymbol{\theta}}_{c}^{\mathrm{T}}, \hat{\boldsymbol{\theta}}_{p}^{\mathrm{T}}\right]^{\mathrm{T}}$ and the corresponding $\hat{\boldsymbol{\theta}}_{m}$ are determined by the best position gbest $^{l_{\max }}$. 


\section{Acknowledgments}

The authors would like to express sincere thanks to Kyushu Electric Power Company, Kagoshima Branch, for offering their data and support in this research.

This work was partially supported by JSPS KAKENHI Grant Number 24560524.

\section{References}

[1] S. Miyazawa: Disaster Prevention and Weather (in Japanese), Asakura Publishing, 1982.

[2] White Paper on Disaster Management 2012 (in Japanese), Cabinet Office, Government of Japan, 2012.

[3] H. Takata, K. Sonoda, T. Hachino and Y. Minari: A prediction method of damage by typhoons to power systems in Kagoshima via linear regression model and neural network (in Japanese), J. of Signal Process., Vol. 3, No. 6, pp. 455461, 1999.

[4] H. Takata and T. Hachino: A prediction method of electric power damage by typhoons in Kagoshima via the secondorder polynomial model and NN (in Japanese), Trans. ISCIE, Vol. 16, No. 10, pp. 513-519, 2003.

[5] H. Takata, K. Komatsu and T. Hachino: Prediction of electric power damage by typhoons in Amami archipelago, J Signal Process., Vol. 9, No. 6, pp. 465-471, 2005.

[6] T. Hachino, H. Asai and H. Takata: Prediction of electric power damage by typhoons in Amami archipelago via Gaussian process model (in Japanese), IEEJ Trans. Electronics, Information and Systems, Vol. 132, No. 12, pp. 1966-1972, 2012.

[7] A. O'Hagan: Curve fitting and optimal design for prediction (with discussion), J. Roy. Statist. Soc. B, Vol. 40, pp. 1-42, 1978.

[8] C. K. I. Williams and C. E. Rasmussen: Gaussian processes for regression, Adv. Neural Inf. Process. Syst., Vol. 8, pp. 514-520, MIT Press, 1996.

[9] C. E. Rasmussen and C. K. I. Williams: Gaussian Processes for Machine Learning, MIT Press, 2006.

[10] J. Kocijan, A. Girard, B. Banko and R. Murray-Smith: Dynamic systems identification with Gaussian processes, Math. Comput. Modell. Dyn. Syst., Vol. 11, No. 4, pp. 411424, 2005

[11] T. Hachino and H. Takata: Identification of continuoustime nonlinear systems by using a Gaussian process model, IEEJ Trans. Electric. Electron. Eng., Vol. 3, No. 6, pp. 620628,2008

[12] A. Girard, C. E. Rasmussen, J. Q. Candela and R. MurraySmith: Gaussian process priors with uncertain inputs application to multiple-step ahead time series forecasting, Adv. Neural Inf. Process. Syst., Vol. 15, pp. 542-552, MIT Press, 2003

[13] S. Brahim-Belhouari and A. Bermak: Gaussian process for nonstationary time series prediction, Comput. Statist. Data Anal., Vol. 47, pp. 705-712, 2004.

[14] T. Hachino and V. Kadirkamanathan: Multiple Gaussian process models for direct time series forecasting, IEEJ Trans. Electric. Electron. Eng., Vol. 6, No. 3, pp. 245-252, 2011.
[15] B. Likar and J. Kocijan: Predictive control of a gas-liquid separation plant based on a Gaussian process model, Comput. Chem. Eng., Vol. 31, No. 3, pp. 142-152, 2007.

[16] Y. Ogura: Science of Weather (in Japanese), Morikita Publishing, 1994.

[17] J. Kennedy and R. C. Eberhart: Particle swarm optimization, Proc. IEEE Int. Conf. Neural Networks, pp. 1942-1948, 1995.

[18] R. von Mises: Mathematical Theory of Probability and Statistics, Academic Press, 1964

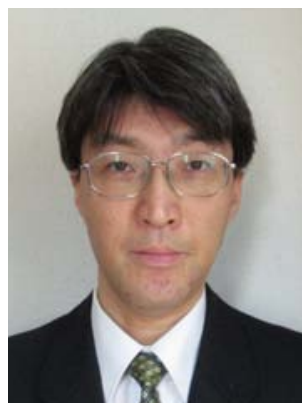

Tomohiro Hachino received his B.S., M.S., and Dr. Eng. degrees in electrical engineering from Kyushu Institute of Technology in 1991, 1993 and 1996, respectively. $\mathrm{He}$ is currently an Associate Professor at the Department of Electrical and Electronics Engineering, Kagoshima University. His research interests include nonlinear control and identification, signal processing, and evolutionary computation. Dr. Hachino is a member of IEEJ, SICE and ISCIE.

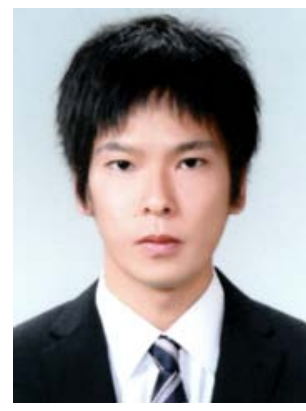

Tatsuya Ueda received his B.S. degree in electrical and electronics engineering from Kagoshima University in 2011. He is currently a master's candidate at the Department of Electrical and Electronics Engineering, Kagoshima University. His research interests include the prediction of nonlinear systems. Mr. Ueda is a student member of IEEJ.

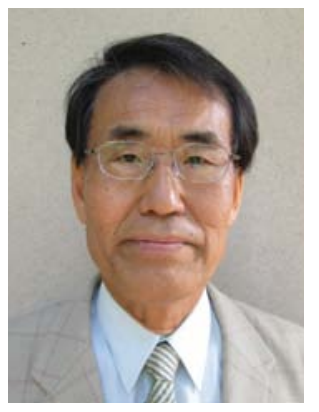

Hitoshi Takata received his B.S. degree in electrical engineering from Kyushu Institute of Technology in 1968 and his M.S. and Dr. Eng. degrees in electrical engineering from Kyushu University in 1970 and 1974, respectively. $\mathrm{He}$ is currently a Professor Emeritus and a part-time lecturer at Kagoshima University. His research interests include the control, linearization, and identification of nonlinear systems.

(Received January 24, 2013; revised April 1, 2013) 